\section{Mit Krautwickel gegen Knieschmerzen}

\section{Krautwickel sind ein altbekanntes Hausmittel bei Arthroseschmerzen. Ob sie tatsächlich die Beschwerden lindern, wurde in Essen überprüft.}

$S_{\mathrm{d} e r}^{\mathrm{el}}$ eit Jahrhunderten wird Weißkraut in der naturheilkundlichen Medizin eingesetzt, etwa als Brustwickel bei stillenden Frauen mit Milchstau. Auch arthrosebedingte Gelenkschmerzen soll ein Wickel aus Weißkrautblättern lindern. Doch wie so oft, fehlen aussagekräftige Studien. Romy Lauche von der Klinik für Naturheilkunde und Integrative Medizin in Essen und ihre Kollegen haben sich des Themas angenommen und die Wirkung des Kohlwickels bei Patienten mit Kniegelenksarthrose Grad II-III (Kellgren-Lawrence-Score) untersucht.

An der Studie teilgenommen haben insgesamt 81 Patienten um die 65 Jahre, die zwischen September 2013 und Juli 2014 in drei Gruppen randomisiert worden waren. Die Patienten der ersten Gruppe (CWL-Gruppe) wurden angehalten, vier Wochen lang einmal täglich für mindestens zwei Stunden oder besser über Nacht am schmerzenden Knie einen Krautwickel aufzulegen. Die Probanden der zweiten Gruppe (TPGGruppe) sollten einmal täglich das Knie mit 1-4 g Diclofenac-haltigem Schmerzgel (10 mg Diclofenac pro g Gel) einreiben und die der dritten Gruppe (UCGruppe) die Behandlung weiterführen, die sie bereits vor der Studie angewandt hatten. Patienten mit sekundär bedingter Arthrose, Patienten, deren Beschwerden mit Kortisoninjektionen behandelt oder die innerhalb der letzten zwölf Monate am Knie operiert worden waren, sowie Patienten mit Leber- und Nierenproblemen, Asthma oder psychischen Erkrankungen waren von der Untersuchung ausgeschlossen. Schwangeren und stillenden Frauen blieb die Teilnahme ebenso verwehrt wie Patienten, die Kortikosteroide bzw. Immunsuppressiva einnahmen oder andere Wickel und $\mathrm{Pa}$ ckungen anwandten.

Hatten die Probanden der drei Gruppen zu Beginn der Studien die Schmerzintensität ähnlich eingeschätzt, etwa bei $40 \mathrm{~mm}$ auf einer visuellen Analogskala von $0-100 \mathrm{~mm}(\mathrm{p}=0,824)$, veränderte sich das im Behandlungsverlauf. Nach vier Wochen gab die Patientengruppe, die Krautwickel aufgelegt hatte, weniger Schmerzen an als die UC-Gruppe (Unterschied -12,1, 95\%-KI -23,1 bis $-1,0 ; \mathrm{p}=0,033)$. Zwischen CWL- und TPG-Gruppe hingegen fand sich kein Unterschied $(-8,6$; $95 \%-\mathrm{KI}-21,5$ bis 4,4 ; $\mathrm{p}=0,190)$. Nach zwölf Wochen waren alle drei Gruppen in puncto Schmerzintensität wieder gleich auf. Bei der Gelenkfunktionalität (WOMAC-Score, 30 sec CST) und Lebensqualität (SF-36) ergab sich für die Krautwickel-Behandlung im Vergleich zur gewohnten Therapie ein klarer Vorteil. Verglichen mit der Schmerzgelgruppe beurteilten die Krautwickel-Patienten nach vier Wochen die Funktionalität des Kniegelen-

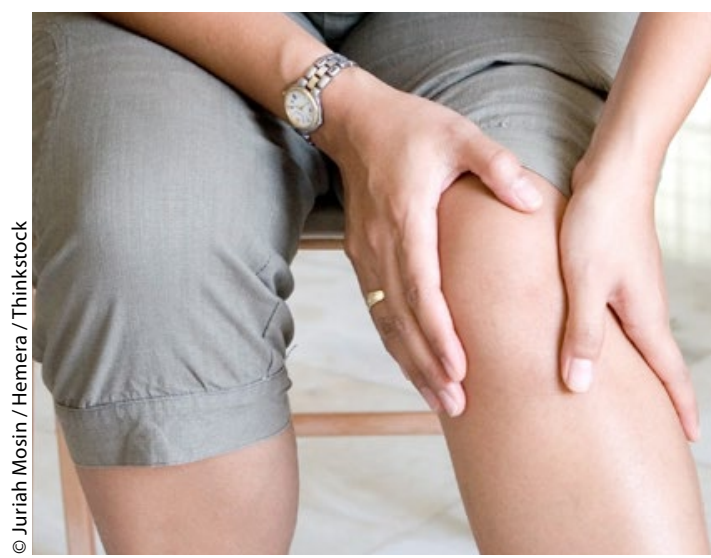

Kraut fürs Knie

kes etwas besser (WOMAC-Index) und nach zwölf Wochen die allgemeine Lebensqualität (SF-36).

Nach einer vierwöchigen Behandlungsdauer schnitt der Krautwickel in puncto Schmerz, Gelenkfunktion und Lebensqualität besser ab als die „vorherige" Therapie. Im Vergleich zur Schmerzgelapplikation zeigte sich aber kein Vorteil, fassen Lauche und ihre Kollegen die Ergebnisse zusammen. Demnach könne, so die Meinung der Studienautoren, diese naturheilkundliche Behandlungsmethode bei Gelenkarthrose guten Gewissens empfohlen werden, aufgrund des guten Sicherheitsprofils auch zur langfristigen Anwendung. Weitere Studien seien aber wünschenswert.

$(d k)$

Lauche R et al. Efficacy of Cabbage Leaf Wraps in the Treatment of Symptomatic Osteoarthritis of the Knee: A Randomized Controlled Trial. Clin J Pain 2016; online 17. Februar; doi: 10.1097/ AJP.0000000000000352

\title{
Schöner leiden mit High Heels
}

\section{Frauen, die auf hohen Absätzen durchs Leben gehen, riskieren Gesundheitsschäden - und das nicht nur bei sich selbst.}

$B^{\text {rimise }}$ ritische Ärzte werteten 18 Studien mit 14.647 Teilnehmerinnen aus. In drei von vier Studien ergab sich ein signifikanter Zusammenhang zwischen Absatzhöhe und Hallux valgus: Der Anteil der Betroffenen unter den HighHeel-Trägern war im Vergleich zu den
Flachbeschuhten um 47-148\% erhöht. Sieben von acht Studien zeigten ein erhöhtes Risiko für Zerrungen, Verstauchungen oder Frakturen von Sprunggelenk oder Fuß durch das Tragen hoher Schuhe. Eine Studie widmete sich den Verletzungen, die Trägerinnen von
High Heels anderen Personen zufügten: 65 Notaufnahme-Vorstellungen wegen solcher Kollateralschäden wurden innerhalb von fünf Jahren im australischen Bundesstaat Victoria gezählt. Meist waren die Opfer versehentlich unter die Absätze geraten, in 16 Fällen waren die High Heels gezielt als Waffe eingesetzt worden.

BMJ Open 2016; 6: e010053 